\title{
Adverse cardiorespiratory events following primary vaccination of very low birth weight infants
}

\author{
C. Meinus, ${ }^{1}$ G. Schmalisch, ${ }^{2}$ S. Hartenstein,${ }^{1}$ H. Proquitté, ${ }^{1}$ C.C. Roehr ${ }^{2}$
}

\begin{abstract}
Objective: To examine the relationship between primary vaccination of preterm infants and prevalence ratios of associated factors for unwanted cardiorespiratory events, following the recommendation of the Centers for Disease Control and Prevention Advisory Committee on Immunization Practices for immunization of preterm infants at 2 months of chronological age.

Methods: Two-year retrospective study of very low birth weight infants receiving their primary vaccination. Major cardiorespiratory events, such as apnea, bradycardia, $\mathrm{SpO}_{2}$ desaturation, and minor adverse events, such as temperature instability, poor handling and local reactions, were recorded. Prevalence ratio with $95 \%$ confidence interval for associated factors between infants with and without cardiorespiratory events was calculated.

Results: Eighty neonates were studied (median [range] birth weight $970 \mathrm{~g}[428-1,490])$, gestational age of 27.4 weeks (23.3-33). Adverse reactions occurred in 35 (44\%) patients: minor events in 19 (24\%), major events in $28(35 \%)$. Infants with major events had significantly lower gestational age $(p=0.008)$ and a higher incidence of bronchopulmonary dysplasia $(71 \%$ vs. $48 \% ; \mathrm{p}<0.05)$. In very low birth weight infants with major events, $\mathrm{O}_{2}$ desaturations before vaccination were 3.40 (1.41-8.23) times higher and treatment with methylxanthines for apnea and bradycardia syndrome was 8.05 (2.50-25.89) times higher compared to infants without major events.

Conclusion: Major cardiorespiratory events occurred in over $1 / 3$ of all very low birth weight infants after vaccination. Associated factors were low gestational age, bronchopulmonary dysplasia, methylxanthine treatment, and persisting $\mathrm{O}_{2}$ desaturations before vaccination. Primary vaccination of very low birth weight infants should be performed under continuous monitoring of vital parameters.
\end{abstract}

J Pediatr (Rio J). 2012;88(2):137-42: Preterm, neonate, very low birth weight, vaccination, adverse event, oxygen saturation, monitoring.

\section{Introduction}

The Centers for Disease Control and Prevention (CDC) Advisory Committee on Immunization Practices (ACIP) recommends immunization of preterm infants at 2 months of chronological age with a diphtheria-tetanus-pertussishaemophilus influenza-polio-hepatitis B and pneumococcal vaccine. ${ }^{1}$ Earlier studies of whole-cell component pertussis vaccine reported on cardiorespiratory events (i.e. recurrence of apnea, bradycardia or $\mathrm{SpO}_{2}$ desaturations) in preterm infants. ${ }^{2-6}$ In recently published studies an acellular pertussis vaccine was used but similar adverse reactions were still being reported. ${ }^{7-12}$ The rate of adverse reactions varied widely between the studies from $6 \%$ to $50 \% .^{2,11-17}$ Studies

1. MD. Department of Neonatology, Charité Universitätsmedizin Berlin, Berlin, Germany.

2. PhD. Department of Neonatology, Charité Universitätsmedizin Berlin, Berlin, Germany.

No conflicts of interest declared concerning the publication of this article.

Suggested citation: Meinus C, Schmalisch G, Hartenstein S, Proquitté H, Roehr CC. Adverse cardiorespiratory events following primary vaccination of very low birth weight infants. J Pediatr (Rio J). 2012;88(2):137-42.

Manuscript submitted Oct 8 2011, accepted for publication Jan 182012.

http://dx.doi.org/10.2223/JPED.2182 
suggest that very low birth weight infants (VLBWI, birth weight $<1,500 \mathrm{~g}$ ) are a particularly vulnerable patient group for developing serious adverse reactions. ${ }^{8,15,16}$ Hacking et al. reviewed a cohort of extremely preterm infants for reactions to primary immunization. ${ }^{17}$ The authors found cardiorespiratory events to be common, the highest incidence being amongst those who had had previous septicemia or a long-standing need for respiratory support. ${ }^{17}$ In contrast, a prospective, randomized controlled study of routine vaccination of VLBWI did not report serious adverse reactions. ${ }^{18}$ Clifford et al. investigated recurrence of apnea after reimmunization through a surveillance system and showed an $18 \%$ recurrence rate for apneas in preterm infants following the second immunization. ${ }^{19}$ Succi et al. recently reviewed the literature on vaccinating infants with special conditions and commented on the very limited available evidence for preterm infants, ${ }^{20}$ warranting an analysis of a large, homogeneous patient group of very low birth weight (VLBW) patients. Therefore, the purpose of this study was to examine the incidence, frequency, severity and types of adverse reactions to vaccination in a large single cohort of VLBWI. Our aim was to identify factors associated with cardiorespiratory reactions following primary vaccination.

\section{Methods}

This is a retrospective analysis of all VLBWI who received their first routine immunization at a tertiary neonatal center, the Department of Neonatology Charité in Berlin, Germany over the period of 24 months (January 2006 to December 2007). During the study period, 151 VLBWI were born alive. All inpatient VLBWI were vaccinated by day 60 of life, according to the current CDC recommendations. ${ }^{1}$ Ethical clearance was obtained from the department prior to the study.

Routine vaccination was carried out after completion of the second month of life, taking into account the infants' clinical condition. The decision to initiate vaccination was made by the attending neonatologist, based on the patient's clinical presentation. All cardiorespiratory stable infants who at the time of vaccination showed no sign of infection received an intramuscular (IM) injection of the vaccine. All infants received either the combined Infanrix (DTPa-IPV) and Hib injection or Infanrix Hexa (DTPa-IPV+Hib+HepB) by GlaxoSmithKline, Munich, Germany, alone or with an additional injection of Prevenar (pneumococcal vaccine) by Wyeth Pharma $\mathrm{GmbH}$, Muenster, Germany.

Recorded data were: birth weight, gestational age (GA) and chronological age, corrected GA, weight at day of vaccination, treatment for apnea or bradycardia at the time of vaccination, treatment for chronic disease (this included pulmonary/cardiac or neurological disease), bronchopulmonary dysplasia (BPD) defined as $\mathrm{O}_{2}$ requirement at day 28 of life, and the need for respiratory support. All infants were under continuous cardiorespiratory monitoring (electrocardiography, and continuous peripheral pulse oximetry), including monitoring of apnea, bradycardia, oxygen saturation, oxygen requirement, and body temperature. The neonatal nursing staff was educated on the nature and presentation of adverse events to immunization. They were instructed to note down any suspected adverse reaction or clinically relevant events from the vital sign monitoring in the patient chart. All case notes were later reviewed with regard to relevant events relating to vaccination, such as episodes of apnea and bradycardia, desaturations, temperature instability, poor feeding or poor handling, and local reactions. To compare pre- and post-vaccination reactions, patient charts from the 48-hour periods preceding and following the vaccinations were analyzed.

Cardiorespiratory symptoms such as apnea, bradycardia or desaturations were considered as major adverse reactions: apnea was defined as $\geq 20$ s without breathing, and bradycardia as a heart rate $\leq 100 / \mathrm{min}$ for at least 20 s. Saturation status was monitored by continuous pulse oximetry. Desaturation was defined as a fall in the peripheral oxygen saturation $\left(\mathrm{SpO}_{2}\right)$ to $85 \%$ or less in the oxygen saturation not attributed to a technical problem or to any other transient cause. Infants were considered to have recurrent or increased cardiorespiratory reactions in case of recurrence of, or a $\geq 50 \%$ increase in the frequency of cardiorespiratory episodes occurred in the 48-hour period following immunization compared to the 48-hour period preceding immunization. Minor adverse reactions were defined as local reactions, fever or poor handling. Fever was defined as a rectal temperature of more than $38.0{ }^{\circ} \mathrm{C}$ during the post-vaccination period in the absence of any other causes. Poor handling was based on the observation of the neonatal nurse looking after the baby and included characteristics such as irritability, feeding intolerance, increasing nasogastric feeds and tachypnea.

To distinguish between infants with reactions secondary to vaccination and those without reactions to vaccination, these two groups were compared with regard to their baseline characteristics and pre-immunization condition. Categorical data were described as $\mathrm{n}(\%)$ and compared by chi-square test or Fisher's exact test as appropriate. Physiologic parameters are presented as median and range and compared by Mann-Whitney rank test. Prevalence ratio (PR) with $95 \%$ confidence intervals (CI) for specific risk factors between infants with and without adverse cardiorespiratory reactions was calculated. Statistical analysis was performed using the Statgraphics Centurion software (version 15.0, Statpoint Inc., Herndon, Virginia, USA). A level of statistical significance of $p<0.05$ was accepted. 


\section{Results}

During the study period, a total of 151 VLBWI were born alive. Of these, 80 VLBWI received their first vaccination while still hospitalized or were rehospitalized for the purpose of primary vaccination. The other 68 VLBWI were discharged prior to vaccination and not readmitted for vaccination. Three infants had to be excluded because they were not on the cardiorespiratory monitoring system for the entire observation period, or documentation of case notes was inadequate. The patients' characteristics are shown in Table 1.

At the time of vaccination 16 (20\%) infants received methylxanthine treatment for apnea and bradycardia syndrome (oral theophylline or caffeine citrate) and 19 (24\%) were receiving other medications, such as diuretics or anticonvulsants. Vaccination was generally administered after the second month of life (i.e. $\geq$ day 60 of life). Eight patients $(10 \%)$ were vaccinated at $<60$ days of life (minimum age 51 days of life) for logistic reasons. Forty $(50 \%)$ patients were vaccinated at $>70$ days of age for clinical reasons such as operations, nosocomial infections, overtly frequent apnea and/or bradycardia; 31 (39\%) patients were vaccinated between $70-99$ days, six $(0.08 \%)$ were vaccinated between $100-119$ days, and three $(0.04 \%)$ were vaccinated after 120 days.

Unwanted adverse reactions to vaccination were noted in $35(44 \%)$ patients. Twenty-eight (35\%) had major cardiorespiratory reactions, and 19 (24\%) had minor reactions. The influence of vaccination was transient; all infants returned to their baseline within 48-72 hours after vaccination. Those who remained stable for 48 hours after immunization did not require any medical intervention at a later time.

Infants showing cardiorespiratory reactions (CREVLBWI) were compared regarding the pre-immunization characteristics to those showing no reactions to vaccination (non-CRE-VLBWI) (Table 2). CRE-VLBWI had a statistically significantly lower gestational $(p=0.008)$ and postconceptional age at immunization $(p=0.05)$ and a higher incidence of BPD ( $71 \%$ vs. $48 \%$; $p<0.05)$, compared to non-CRE-VLBWI.

Prevalence of diagnoses and treatments of infants with and without adverse cardiorespiratory events is shown in Table 3. In CRE-VLBWI desaturations were 3.40 (1.41-8.23) times higher and the treatment with methylxanthine for apnea and bradycardia was 8.05 (2.50-25.89) times higher compared to non-CRE-VLBWI. Although prevalence of respiratory support in CRE-VLBWI was 5.57 (0.61-51.1) times higher compared to non-CRE-VLBWI, PR was not statistically significant due to the low total number of infants on respiratory support in our cohort (4/80).

\section{Discussion}

We studied the occurence of post-vaccination reactions in VLBWI to analyze the incidence of significant unwanted and adverse reactions to vaccination in our patient population, and to identify associated factors for significant adverse reactions in this high-risk patient group. In our study, the incidence of cardiorespiratory reactions in the

Table 1 - Characteristics of the study population: data given as median (range) or $\mathrm{n}(\%)$

\begin{tabular}{|c|c|c|c|}
\hline Variable & n (\%) & Median & Range \\
\hline Gestational age (weeks) & & 27.36 & $23.3-33.0$ \\
\hline Birth weight (g) & & 970 & $428-1,490$ \\
\hline Male & $40(50 \%)$ & & \\
\hline Multiple birth & $26(32.5 \%)$ & & \\
\hline Apgar 5 min. & & 7 & $3-10$ \\
\hline BPD & $45(56 \%)$ & & \\
\hline IVH & $19(24 \%)$ & & \\
\hline PVL & $5(6 \%)$ & & \\
\hline \multicolumn{4}{|l|}{ Time of immunization } \\
\hline Postconceptional age (weeks) & & 37.29 & $32.6-47.2$ \\
\hline Chronological age (weeks) & & 69.5 & 51-136 \\
\hline Weight $(g)$ & & $2,072.5$ & $1,205-3,800$ \\
\hline Respiratory support & $4(5 \%)$ & & \\
\hline Treatment for chronic disease & $19(24 \%)$ & & \\
\hline Treatment for apnea and bradycardia & $16(20 \%)$ & & \\
\hline
\end{tabular}

$\mathrm{BPD}=$ bronchopulmonary dysplasia; IVH = intraventricular hemorrhage; PVL = periventricular leucomalacia. 
Table 2 - Patient characteristics of infants with and without adverse cardiorespiratory events following vaccination: data given are median (range) or $\mathrm{n}(\%)$

\begin{tabular}{|c|c|c|c|}
\hline & $\begin{array}{c}\text { CRE } \\
n=28\end{array}$ & $\begin{array}{c}\text { Non-CRE } \\
n=52\end{array}$ & p value \\
\hline Gestational age (weeks) & $26(23-31)$ & $28(23-33)$ & $0.008 *$ \\
\hline Birth weight $(g)$ & $815(550-1,370)$ & $980(428-1,490)$ & 0.138 \\
\hline Male & $14 / 28(50 \%)$ & $26 / 52(50 \%)$ & 1.000 \\
\hline Postconceptional age at time of vaccination (weeks) & $37(33-42)$ & $38(34-47)$ & 0.160 \\
\hline Chronological age at time of vaccination (weeks) & $74(58-104)$ & $64(51-136)$ & 0.897 \\
\hline Body weight at time of vaccination $(\mathrm{g})$ & $2,032(1,205-2,900)$ & $2,155(1,400-3,800)$ & 0.168 \\
\hline
\end{tabular}

$\mathrm{CRE}=$ infants with cardiorespiratory event; non-CRE = infants without cardiorespiratory event.

* Significant.

Table 3 - Prevalence of diagnoses and treatments before vaccination of infants with and without adverse cardiorespiratory events: data given are $\mathrm{n}(\%)$ and prevalence ratio with $95 \%$ confidence interval

\begin{tabular}{|c|c|c|c|c|}
\hline & $\begin{array}{c}\text { CRE } \\
n=28\end{array}$ & $\begin{array}{c}\text { Non-CRE } \\
n=52\end{array}$ & PR with $95 \%$ CI & p value \\
\hline Apnea or bradycardia & $6 / 28(21 \%)$ & $5 / 52(10 \%)$ & $1.22(0.75-6.66)$ & 0.143 \\
\hline Chronic $\mathrm{O}_{2}$ desaturations & $11 / 28(39 \%)$ & $6 / 52(12 \%)$ & $3.40(1.41-8.23)$ & $0.004 *$ \\
\hline Respiratory support & $3 / 28(11 \%)$ & $1 / 52(2 \%)$ & $5.57(0.61-51.1)$ & 0.121 \\
\hline Methylxanthine treatment & $13 / 28(46 \%)$ & $3 / 52(6 \%)$ & $8.05(2.50-25.89)$ & $<0.001^{*}$ \\
\hline BPD & $20 / 28(71 \%)$ & $25 / 52(48 \%)$ & $1.49(1.03-2.14)$ & $0.045^{*}$ \\
\hline IVH & $5 / 28(18 \%)$ & $14 / 52(27 \%)$ & $0.66(0.27-1.65)$ & 0.363 \\
\hline
\end{tabular}

$95 \% \mathrm{Cl}=95 \%$ confidence interval; BPD = bronchopulmonary dysplasia; CRE = infants with cardiorespiratory event; IVH = intraventricular hemorrhage; non-CRE = infants without cardiorespiratory event; $\mathrm{PR}=$ prevalence ration

* Significant.

post-vaccination period was 35\%. Lower GA and clinical signs of BPD were significant risk factors for vaccinerelated cardiorespiratory reactions. The prevalence of persistence of $\mathrm{O}_{2}$ desaturations and of treatment with methylxanthines for apnea and bradycardia syndrome before vaccination was statistically significantly higher in CRE-VLBWI compared to non-CRE-VLBWI. Fortunately, the majority of reactions were mild and transient. However, there were also severe cardiorespiratory reactions, which required vigorous stimulation, oxygen supplementation and once brief use of manual ventilation support. Analysis of associated factors for cardiorespiratory reactions in the post-immunization period, as performed in several other retrospective studies, came to similar observations. $5-9,15-18$ Recurrence of apnea after the second vaccination has been reported to be $18 \%$ in preterm infants. ${ }^{19}$ Hence, recognition of associated factors for severe cardiorespiratory reactions in the post-immunization period, as identified in our study, is of special importance for those infants discharged from the hospital before the time of vaccination or those debating readmission to hospital for the vaccination. ${ }^{16-19}$

Following our data analysis and the results of the above studies, we suggest that: a) VLBWI should be closely monitored during their first vaccination as inpatients; b) those with reactions to the primary vaccination should be readmitted for the second immunization; and c) occurrence of and risk factors for adverse reactions to vaccination of VLBWI should be investigated in sufficiently large scale, prospective randomized controlled trials (RCT).

Reflecting the current state of evidence, the CDC and also the German Academy for Pediatrics now recommend hospitalization for the first immunization (acellular 
diphtheria-pertussis-tetanus, haemophilus influenza type $B$, hepatitis $B$ and pneumococcal vaccine) for infants born at, or less than 28 weeks of GA. 21,22

Our study has a number of limitations. We performed a retrospective analysis of a large body of VLBWI as a preliminary investigation to plan a prospective trial. Retrospective analysis allows no control of qualitative or quantitative monitoring. The evaluated data was based on the documentation by staff, who may have had a variable threshold to manually mark cardiorespiratory reactions in the charts despite specific internal nursery protocol. A prospective, possibly blinded protocol would help exclude such observer-specific confounders. As advised by the $\mathrm{CDC}$, the vaccinations were performed after day 60 of life.1,22 Few vaccinations (10\%) were advanced before this date to facilitate early discharge from hospital. Others (50\%) were delayed to a later postnatal date, mainly for clinical reasons. Both situations could have biased our results. Routine immunization on a fixed date without clinical reasoning would not be prudent. The varying clinical conditions on day 60 of life could have a particular and profound impact on the reactogenicity to the vaccination. ${ }^{12}$

Demirjian and Levy reviewed the safety and efficacy of neonatal vaccinations. ${ }^{23}$ According to the authors, immunological immaturity is key when expecting adverse reactions to immunization and the more preterm the infants, the less developed their immune system will be. In addition, Day et al. showed that alum- or lipid-adjuvanted vaccines induce greater tissue inflammation than non-adjuvanted vaccines. ${ }^{24}$ Adverse reactogenicity to immunization before day 60 of age may therefore be due to a less mature immune system, leaner body composition, and possibly increased systemic sensitivity to local inflammatory reactions. Wood and Siegrist concluded that until further evidence is reached, many hurdles to neonatal vaccination remain, including immunological and clinical safety concerns. ${ }^{25}$ Accordingly, vaccination before day 60 is not encouraged by the CDC. ${ }^{1,22}$

We conclude that adverse cardiorespiratory events to primary immunization are common in VLBW preterm infants. Important risk factors for significant postimmunization cardiorespiratory reactions are low GA, BPD and methylxanthine therapy for apnea and bradycardia syndrome, and chronic $\mathrm{O}_{2}$ desaturations before vaccination. However, despite the identification of predisposing factors for adverse events it remains difficult to predict which VLBWI will show an adverse reaction to immunization, and at which intensity. Thus, we suggest that all VLBW neonates should be closely monitored over 48 hours following the first immunization, preferably as inpatients.

\section{References}

1. Centers for Disease Control and Prevention (CDC). Updated recommendations for use of tetanus toxoid, reduced diphtheria toxoid and acellular pertussis vaccine (Tdap) in pregnant women and persons who have or anticipate having close contact with an infant aged < 12 months - Advisory Committee on Immunization Practices (ACIP), 2011. MMWR Morb Mortal Wkly Rep. 2011;60:1424-6.

2. Botham SJ, Isaacs D. Incidence of apnoea and bradycardia in preterm infants following triple antigen immunization. J Paediatr Child Health. 1994;30:533-5.

3. Cooper PA, Madhi SA, Huebner RE, Mbelle N, Karim SS, Kleinschmidt $I$, et al. Apnea and its possible relationship to immunization in ex-premature infants. Vaccine. 2008;26:3410-3.

4. Ramsay ME, Miller E, Ashworth LA, Coleman TJ, Rush M, Waight PA. Adverse events and antibody response to accelerated immunisation in term and preterm infants. Arch Dis Child. 1995;72:230-2.

5. Sánchez PJ, Laptook AR, Fisher L, Sumner J, Risser RC, Perlman JM. Apnea after immunization of preterm infants. J Pediatr. 1997; 130:746-51.

6. Sen S, Cloete Y, Hassan K, Buss P. Adverse events following vaccination in premature infants. Acta Paediatr. 2001;90:916-20.

7. Faldella G, Galletti S, Corvaglia L, Ancora G, Alessandroni R. Safety of DTaP-IPV-HIb-HBV hexavalent vaccine in very premature infants. Vaccine. 2007;25:1036-42.

8. Flatz-Jequier A, Posfay-Barbe KM, Pfister RE, Siegrist CA. Recurrence of cardiorespiratory events following repeat DTaPbased combined immunization in very low birth weight premature infants. J Pediatr. 2008;153:429-31.

9. Omeñaca F, Garcia-Sicilia J, García-Corbeira P, Boceta R, Romero A, Lopez $\mathrm{G}$, et al. Response of preterm newborns to immunization with ahexavalent diphtheria-tetanus-acellular pertussis-hepatitis B virus-inactivated polio and Haemophilus influenzae type bvaccine: first experiences and solutions to a serious and sensitive issue. Pediatrics. 2005;116:1292-8.

10. Pfister RE, Aeschbach V, Niksic-Stuber V, Martin BC, Siegrist CA. Safety of DTaP-based combined immunization in very-lowbirth-weight premature infants: frequent but mostly benign cardiorespiratory events. J Pediatr. 2004;145:58-66.

11. Schulzke $S$, Heininger $U$, Lücking-Famira $M$, Fahnenstich $H$. Apnoea and bradycardia in preterm infants following immunisation with pentavalent or hexavalent vaccines. Eur J Pediatr. 2005;164:432-5.

12. David S, Vermeer-de Bondt PE, van der Maas NA. Reactogenicity of infant whole cell pertussis combination vaccine compared with acellular pertussis vaccines with or without simultaneous pneumococcal vaccine in the Netherlands. Vaccine. 2008;26:5883-7.

13. Botham SJ, Isaacs $D$, Henderson-Smart DJ. Incidence of apnoea and bradycardia in preterm infants following DTPW and Hib immunization: a prospective study. J Paediatr Child Health. 1997;33:418-21.

14. Ellison VJ, Davis PG, Doyle LW. Adverse reactions to immunization with newer vaccines in the very preterm infant. J Paediatr Child Health. 2005;41:441-3.

15. Klein NP, Massolo ML, Greene J, Dekker CL, Black S, Escobar GJ, et al. Risk factors for developing apnea after immunization in theneonatal intensive care unit. Pediatrics. 2008;121:463-9.

16. Lee J, Robinson JL, Spady DW. Frequency of apnea, bradycardia, and desaturations following first diphtheria-tetanus-pertussisinactivated polio-Haemophilus influenzae type B immunization in hospitalized preterm infants. BMC Pediatr. 2006;6:20.

17. Hacking DF, Davis PG, Wong E, Wheeler K, McVernon J. Frequency of respiratory deterioration after immunisation in preterm infants. J Paediatr Child Health. 2010;46:742-8. 
18. Carbone T, McEntire B, Kissin D, Kelly D, Steinschneider A, Violaris $\mathrm{K}$, et al. Absence of an increase in cardiorespiratory events after diphtheria-tetanus-acellular pertussis immunization in preterm infants: a randomized, multicenter study. Pediatrics. 2008; 121:e1085-90.

19. Clifford V, Crawford NW, Royle J, Lazzaro T, Danchin M, Perrett $\mathrm{KP}$, et al. Recurrent apnoea post immunisation: Informing reimmunisationpolicy. Vaccine. 2011;29:5681-7.

20. Succi RC, Farhat CK. Vaccination in special situations. J Pediatr (Rio J). 2006;82:S91-100.

21. Heininger $U$, Bartmann P, Huppertz HI, Kinet M, Klein R, Korenke C. Respiratory monitoring in former premature infants $(<28$ GW) following primary immunization. Monatsschr Kinderheilkd. 2009;157:173-6.

22. National Center for Immunization and Respiratory Diseases. General recommendations on immunization - recommendations of the Advisory Committee on Immunization Practices (ACIP). MMWR Recomm Rep. 2011;60:1-64.
23. Demirjian A, Levy O. Safety and efficacy of neonatal vaccination. Eur J Immunol. 2009;39:36-46.

24. Day MJ, Schoon HA, Magnol JP, Saik J, Devauchelle P, Truyen U, et al. A kinetic study of histopathological changes in the subcutis of cats injected with non-adjuvanted and adjuvanted multicomponent vaccines. Vaccine. 2007;25:4073-84.

25. Wood N, Siegrist CA. Neonatal immunization: where do we stand? Curr Opin Infect Dis. 2011;24:190-5.

Correspondence:

Dr. Charles Christoph Roehr

Department of Neonatology - Charité Universitätsmedizin Berlin

Chariteplatz 1, 10117 - Berlin - Germany

Fax: +49 (30) 450516920

Tel.: +49 (30) 450516052

E-mail: christoph.roehr@charite.de 